\title{
On certain maximal cyclic modules for the quantized special linear algebra at a root of unity *
}

\author{
KAnEDA Masaharu \\ 558-8585 Osaka, Japan \\ Osaka City University \\ Department of Mathematics \\ kaneda@sci.osaka-cu.ac.jp
}

\author{
Nakashima Toshiki \\ 102-8554 Tokyo, Japan \\ Sophia University \\ Department of Mathematics \\ toshiki@mm.sophia.ac.jp
}

October 26, 2018

\begin{abstract}
By properly specializing the parameters irreducible modules of maximal dimension for the De Concini-Kac version of the Drinfeld-Jimbo quantum algebra in type $A$ may be transformed into modules over Lusztig's infinitesimal quantum algeba. Thus obtained modules have a simple socle and a simple head, and share the same dimension as the infinitesimal Verma modules. Despite these common features we find that they are never isomorphic to infinitesimal Verma modules unless they are irreducible. The same carry over to the modular setup for the special linear groups in positive characteristic.
\end{abstract}

The finite dimensional irreducible representations of the De Concini-Kac version of the Drinfeld-Jimbo quantized enveloping algebra at a complex $\ell$-th root of 1 have their dimensions bounded above, generically attaining a maximal dimension [DK]. In type A Date, Jimbo, Miki and Miwa [DJMM] have given a concrete realization of most of those of maximal dimension. By properly specializing their parametres the second named author of the present paper found in $\mathbb{N}$ that they afford modules $\mathcal{V}$, rarely irreducible, for Lusztig's "infinitesimal" quantum algebra $\mathfrak{u}$ [L1] and that each $\mathcal{V}$ has a unique, up to scalar, invariant vector $u_{\overrightarrow{0}}$ relative to a Borel subalgebra $\mathfrak{u}^{\sharp}$ of $\mathfrak{u}$, and hence that $\mathcal{V}$ has a simple socle generated by $u_{\overrightarrow{0}}$. The dimension being right, it is tempting to compare $\mathcal{V}$ with Humphreys' "infinitesimal" Verma modules [H] quantized by Andersen, Polo and Wen APW], which are the standard objects of study in the representation theory of $\mathfrak{u}$.

It is easy to see that $\mathcal{V}$ is isomorphic to an infinitesimal Verma module as $\mathfrak{u}^{\sharp}$-module, which in turn shows that $\mathcal{V}$ has the same simple head as the infinitesimal Verma module. The explicit description of the actions of the standard generators of $\mathfrak{u}$ on $\mathcal{V}$ allows us, however, to find that $\mathcal{V}$ has also a unique, up to scalar, invariant vector $u_{-}$with respect to the opposite infinitesimal Borel subalgebra. It follows that $\mathcal{V}$ does not lift to an integrable $\mathfrak{u} U_{\mathbb{C}}^{0}$-module, and hence $\mathcal{V}$ can not be isomorphic to any infinitesimal Verma module as $\mathfrak{u}$-module unless $\mathcal{V}$ is simple, where $U_{\mathbb{C}}^{0}$ is the Cartan part of Lusztig's quantum algebra $U_{\mathbb{C}}$ at the $\ell$-th root of 1 .

*supported in part by JSPS Grants in Aid for Scientific Research 
By construction $\mathcal{V}$ may be defined over $\mathcal{B}=\mathbb{Z}\left[v, v^{-1}\right] /\left(\phi_{\ell}\right)$ with $\phi_{\ell}$ the $\ell$-th cyclotomic polynomial in indeterminate $v$. If $\ell$ is an odd prime $p, \mathcal{B} / p \mathcal{B}$ is a finite field $\mathbb{F}_{p}$ of $p$ elements. Let $G$ be the special linear group scheme over $\mathbb{F}_{p}$ with opposite Borel subgroups $B$ and $B^{+}$, and let $G_{1}, B_{1}, B_{1}^{+}$be the Frobenius kernel of $G, B$, and $B_{1}^{+}$, respectively. If $\mathcal{V}_{\mathcal{B}}$ is the $\mathcal{B}$-form of $\mathcal{V}$, then $\mathcal{V}_{p}=\mathcal{V}_{\mathcal{B}} \otimes_{\mathcal{B}} \mathbb{F}_{p}$ is naturally a $G_{1}$-module. We find that $u_{\overrightarrow{0}} \otimes 1$ (resp. $u_{-} \otimes 1$ ) remains a unique, up to $\mathbb{F}_{p}^{\times}, B_{1}^{+}$- (resp. $B_{1^{-}}$)invariant vector in $\mathcal{V}_{p}$. Hence $\mathcal{V}_{p}$ is isomorphic to an infinitesimal Verma module as $B_{1}^{+}$-module, but not as $G_{1}$-module unless $\mathcal{V}_{p}$ is simple.

If the simple $\mathfrak{u}$-module generated by $u_{\overrightarrow{0}}$ in $\mathcal{V}$ and the simple $G_{1}$-module generated by $u_{\overrightarrow{0}} \otimes 1$ in $c V_{p}$ have the same dimension, Lusztig's conjecture for the irreducible characters of $G$-modules will follow from the celebrated theorems of Kazhdan and Lusztig [KL and Kashiwara and Tanisaki [KT] and Casian [Q].

If $\mathcal{C}$ is a category, $\mathcal{C}(A, B)$ will denote the set of morphisms of $\mathcal{C}$ from object $X$ of $\mathcal{C}$ to object $Y$ of $\mathcal{C}$. If $A$ is a ring, $A$ Mod will denote the category of left $A$-modules.

We are grateful to H.H. Andersen and J.C. Jantzen for a helpful comment.

\section{$1^{\circ}$ Infinitesimal Verma modules}

In this section we recollect some facts about infinitesimal Verma modules over an arbitrary quantum algebra of finite type.

(1.1) Let $\mathbb{Q}(v)$ be the fractional field of the Laurent polynomial $\operatorname{ring} \mathcal{A}=\mathbb{Z}\left[v, v^{-1}\right]$ in indeterminate $v, A=\left[\left(A_{i j}\right)\right]$ an indecomposable Cartan matrix of finite type and let $\mathbf{U}$ be the associated Drinfeld-Jimbo quantum algebra over $\mathbb{Q}(v)$ with generators $E_{i}, F_{i}$, and $K_{i}^{ \pm 1}, i \in[1, n]$. Let $U$ be Lusztig's $\mathcal{A}$-subalgebra of $\mathbf{U}$ generated by $E_{i}^{(r)}=\frac{E_{i}^{r}}{[r]_{i} !}, F_{i}^{(r)}=\frac{F_{i}^{r}}{[r]_{i} !}$, $K_{i}^{ \pm 1}, i \in[1, n], r \in \mathbb{N}$, where $[r]_{i} !=\prod_{s=1}^{r}[s]_{i}$ with $[s]_{i}=\frac{v_{i}^{s}-v_{i}^{-s}}{v_{i}-v_{i}^{-1}}, v_{i}=v^{d_{i}}, d_{i} \in\{1,2,3\}$ minimal such that the matrix $\left[\left(d_{i} A_{i j}\right)\right]$ is symmetric.

Let $R$ (resp. $\Lambda$ ) be the root system (resp. the weight lattice) associated to $A$ and $R^{+}$ a positive subsystem of $R$ with the simple roots $\alpha_{i}, i \in[1, n]$. We equip $\Lambda$ with a partial order defined by $R^{+}$as usual. Let $\Lambda^{\vee}$ be the colattice of $\Lambda$ and denote by $\langle\rangle:, \Lambda \times \Lambda^{\vee} \rightarrow \mathbb{Z}$ the perfect pairing. If $\alpha \in R$, let $\alpha^{\vee}$ be its coroot. We set $\lambda_{i}=\left\langle\lambda, \alpha_{i}^{\vee}\right\rangle$ for $\lambda \in \Lambda$. Let

$U^{0}$ be the $\mathcal{A}$-subalgebra of $U$ generated by $K_{i}^{ \pm 1}$ and $\left[\begin{array}{c}K_{i} ; c \\ r\end{array}\right]=\prod_{s=1}^{r} \frac{K_{i} v_{i}^{c-s+1}-K_{i}^{-1} v_{i}^{-c+s-1}}{v_{i}^{s}-v_{i}^{-s}}$, $i \in[1, n], c \in \mathbb{Z}, r \in \mathbb{N}$. Each $\lambda \in \Lambda$ defines an $\mathcal{A}$-algebra homomorphism $\chi_{\lambda}: U^{0} \rightarrow \mathcal{A}$ such that

$$
K_{i} \mapsto v_{i}^{\lambda_{i}}, \quad\left[\begin{array}{c}
K_{i} ; c \\
r
\end{array}\right] \mapsto\left[\begin{array}{c}
\lambda_{i}+c \\
r
\end{array}\right]_{i}=\frac{\prod_{s=0}^{r-1}\left[\lambda_{i}+c-s\right]_{i}}{[r]_{i} !} \quad \forall i \in[1, n], c \in \mathbb{Z}, r \in \mathbb{N} .
$$

(1.2) Let $\ell$ be a positive integer greater than 2 prime to all entries $A_{i j}$ of the Cartan matrix $A, \mathcal{K}=\mathbb{Q}[v] /\left(\phi_{\ell}\right)$, and set $U_{\mathcal{K}}=U \otimes_{\mathcal{A}} \mathcal{K}$. Let $\mathfrak{u}\left(\right.$ resp. $\left.\mathfrak{u}^{+} ; \mathfrak{u}^{-} ; \mathfrak{u}^{0}\right)$ be the $\mathcal{K}$-subalgebra 
of $U_{\mathcal{K}}$ generated by $E_{i} \otimes 1, F_{i} \otimes 1, K_{i} \otimes 1$ (resp. $\left.E_{i} \otimes 1 ; F_{i} \otimes 1 ; K_{i} \otimes 1\right), i \in[1, n]$. Let also $\mathfrak{u}^{\sharp}=\mathfrak{u}^{+} \mathfrak{u}^{0}$ and $\mathfrak{u}^{b}=\mathfrak{u}^{0} \mathfrak{u}^{-}$. We will abbreviate $x \otimes 1$ of $U_{\mathcal{K}}$ as $x$, and $\chi_{\lambda} \otimes_{\mathcal{A}} \mathcal{K}$ as $\chi_{\lambda}$.

Let $\tilde{\mathfrak{u}}$ be the $\mathcal{K}$-subalgebra of $U_{\mathcal{K}}$ generated by $\mathfrak{u}$ and $U_{\mathcal{K}}^{0}=U^{0} \otimes_{\mathcal{A}} \mathcal{K}$, and let $\tilde{\mathfrak{u}}^{\sharp}=\mathfrak{u}^{\sharp} U_{\mathcal{K}}^{0}$, $\tilde{\mathfrak{u}}^{b}=U_{\mathcal{K}}^{0} \mathfrak{u}^{b}$. Each $\lambda \in \Lambda$ defines a 1-dimensional $\tilde{\mathfrak{u}}^{b}$-module by $\chi_{\lambda}$ annihilating all $F_{i}$, which we will still denote by $\lambda$. Let $\tilde{\nabla}(\lambda)=\tilde{\mathfrak{u}}^{b} \operatorname{Mod}(\tilde{\mathfrak{u}}, \lambda)$. We make $\tilde{\nabla}(\lambda)$ into a $\tilde{\mathfrak{u}}$-module by setting $x f=f(? x)$ for each $x \in \tilde{\mathfrak{u}}$ and $f \in \tilde{\nabla}(\lambda)$.

Let $\Lambda^{\text {res }}=\left\{\nu \in \Lambda \mid \nu_{i} \in[0, \ell-1] \forall i\right\}$. If we write $\lambda=\lambda^{0}+\ell \lambda^{1}$ with $\lambda^{0} \in \Lambda^{\text {res }}$ and $\lambda^{1} \in \Lambda$, one has from [APW, 1.8] an isomorphism of $\tilde{\mathfrak{u}}$-modules

$$
\tilde{\nabla}(\lambda) \simeq \tilde{\nabla}\left(\lambda^{0}\right) \otimes_{\mathcal{K}} \ell \lambda^{1}
$$

where $\ell \lambda^{1}$ ia a 1 -dimensional $\tilde{\mathfrak{u}}$-module defined by $\chi_{\ell \lambda^{1}}$ annihilating all $E_{i}, F_{i}$ and $K_{i}-1$.

On the other hand, the natural gradation on $\mathfrak{u}^{+}$assigning each $E_{i}$ grade $\alpha_{i}$ equips $\mathfrak{u}^{+}$ with a structure of $\tilde{\mathfrak{u}}^{\sharp}$-module such that $\mathfrak{u}^{+}$act by the left multiplication and $U_{\mathcal{K}}^{0}$ by $\chi_{-\lambda+\nu}$ on the $\nu$-th homogeneous part of $\mathfrak{u}^{+}, \nu \in \sum_{i} \mathbb{N} \alpha_{i}$. Recall antiautomorphism $\Psi$ on $U$ such that $E_{i} \mapsto E_{i}, F_{i} \mapsto F_{i}$ and $K_{i} \mapsto K_{i}^{-1}, i \in[1, n]$. If $M$ is a $\tilde{\mathfrak{u}}^{\sharp}$-module of finite type, we will denote by $M^{\Psi}$ the $\mathcal{K}$-linear dual of $M$ made into $\tilde{\mathfrak{u}}^{\sharp}$-module by setting $x f=f(\Psi(x)$ ?) for each $x \in \tilde{u}^{\sharp}, f \in \operatorname{Mod}_{\mathcal{K}}(M, \mathcal{K})$. Then we have an isomorphism of $\tilde{u}^{\sharp}$-modules

$$
\tilde{\nabla}(\lambda) \simeq\left(\mathfrak{u}^{+}\right)^{\Psi} \quad \text { via } \quad f \mapsto f \circ\left(\Psi \otimes_{\mathcal{A}} \mathcal{K}\right) .
$$

One can likewise define a $\mathfrak{u}$-module $\nabla(\lambda)=\mathfrak{u}^{b} \operatorname{Mod}(\mathfrak{u}, \lambda)$. By restricting the $\tilde{\mathfrak{u}}$-action to $\mathfrak{u}, \tilde{\nabla}(\lambda)$ yields $\nabla(\lambda)$. Then the isomorphism (2) restricts to an isomorphism of $\mathfrak{u}^{\sharp}$-modules

$$
\nabla(\lambda) \simeq\left(\mathfrak{u}^{+}\right)^{\Psi}
$$

(1.3) Recall from Xi $₫, 2.5]$ that

$$
\mathfrak{u}^{+} \text {has a simple socle } \mathcal{K} \prod_{\alpha \in R^{+}} E_{\alpha}^{\ell-1} \text { as } \mathfrak{u}^{+} \text {-module, }
$$

where $E_{\alpha}$ is a root vector of $\mathfrak{u}^{+}$associated to $\alpha \in R^{+}[\mathrm{L} 2]$ and the product is taken in a certain specific order. It follows that $\mathfrak{u}^{+}$is indecomposable as $\mathfrak{u}^{+}$-module, and hence that

$$
\mathfrak{u}^{+} \text {is a projective cover of trivial module } \mathcal{K} \text { as } \mathfrak{u}^{+} \text {-module. }
$$

By an integrable $\tilde{\mathfrak{u}}$ - (resp. $\mathfrak{u}$-) module $M$ we will mean a $\tilde{\mathfrak{u}}$ - (resp. $\mathfrak{u}$-) module $M$ such that

$$
M=\coprod_{\mu \in \Lambda} M_{\mu}, \quad \text { with } \quad M_{\mu}=\left\{m \in M \mid t m=\chi_{\mu}(t) m \forall t \in U_{\mathcal{K}}^{0}\left(\text { resp. } \mathfrak{u}^{0}\right)\right\} .
$$

Each $\tilde{\nabla}(\lambda)$ (resp. $\nabla(\lambda)$ ) is an integrable $\tilde{\mathfrak{u}}$ - (resp. $\mathfrak{u}$-) module. Define integrable $\tilde{\mathfrak{u}}^{\sharp}$ - and $\mathfrak{u}^{\sharp}$-modules likewise. One obtains from $(2)$ and $(1.2 .2,3)$

Proposition. For each $\lambda \in \Lambda$ the $\mathfrak{u}^{\sharp}$ (resp. $\tilde{\mathfrak{u}}^{\sharp}$ ) module $\nabla(\lambda)$ (resp. $\tilde{\nabla}(\lambda)$ ) is an injective hull of $\lambda$ in the category of integrable $\mathfrak{u}^{\sharp}$ - (resp. $\tilde{\mathfrak{u}}^{\sharp}$-) modules. 
(1.4) Let $\zeta$ be the image of $v$ in $\mathcal{K}$, and let $U_{\zeta}$ be the De Concini-Kac algebra [DK] over $\mathcal{K}$ associated to the Cartan matrix $A$ with the generators $E_{i}, F_{i}, K_{i}^{ \pm 1}, i \in[1, n]$, and the same relations for $\mathbf{U}$ with $v$ replaced by $\zeta$. For each $\alpha \in R^{+}$let $E_{\alpha}$ (resp. $F_{\alpha}$ ) be the root vector of $U_{\zeta}$ associated with $\alpha$ (resp. $-\alpha$ ), and let $\bar{U}_{\zeta}=U_{\zeta} /\left(E_{\alpha}^{\ell}, F_{\alpha}^{\ell} \mid \alpha \in R^{+}\right)$. If $\bar{U}_{\zeta}^{b}$ is the $\mathcal{K}$-subalgebra of $\bar{U}_{\zeta}$ generated by all $F_{\alpha}, \alpha \in R^{+}$, and $K_{i}, i \in[1, n]$, each $\lambda \in \Lambda$ defines a 1-dimensional $\bar{U}_{\zeta}^{b}$-module by annihilating all $F_{\alpha}$ and letting $K_{i}$ act by $\zeta^{d_{i} \lambda_{i}}$. Then $\bar{U}_{\zeta} \otimes_{\bar{U}_{\zeta}^{b}} \lambda$ comes equipped with a structure of $\tilde{\mathfrak{u}}$-module [AJS, 2.10] such that each $x \in U_{\mathcal{K}}^{0}$ acts on $\prod_{\alpha \in R^{+}} E_{\alpha}^{c_{\alpha}} \otimes 1, c_{\alpha} \in \mathbb{N}$, by the scalar $\chi_{\lambda+\sum_{\alpha} c_{\alpha} \alpha}(x)$. Put $\rho=\frac{1}{2} \sum_{\alpha \in R^{+}} \alpha$.

Proposition. For each $\lambda \in \Lambda$ we have an isomorphism of $\tilde{\mathfrak{u}}$-modules

$$
\tilde{\nabla}(\lambda) \simeq \bar{U}_{\zeta} \otimes_{\bar{U}_{\zeta}^{b}}(\lambda-2(\ell-1) \rho)
$$

Proof: Let $\varepsilon_{\lambda} \in \tilde{\nabla}(\lambda)$ be the element induced by the counit of $\mathfrak{u}^{+}$. By the universality of $\tilde{\nabla}(\lambda)[$ APW, $0.8 .1]$ there is a homomorphism of $\tilde{\mathfrak{u}}$-modules

$$
\bar{U}_{\zeta} \otimes_{\bar{U}_{\zeta}^{b}}(\lambda-2(\ell-1) \rho) \rightarrow \tilde{\nabla}(\lambda) \text { such that } \quad E^{+} \otimes 1 \mapsto \varepsilon_{\lambda},
$$

where $E^{+}=\prod_{\alpha \in R^{+}} E_{\alpha}^{\ell-1}$. On the other hand, by [AJS, 4.9]

$$
\text { the } \tilde{\mathfrak{u}} \text {-socle of } \bar{U}_{\zeta} \otimes_{\bar{U}_{\zeta}^{b}}(\lambda-2(\ell-1) \rho) \text { is simple of highest weight } \lambda \text {. }
$$

It follows that the map (1) is injective, and hence bijective by dimension.

(1.5) Corollary. Each $\tilde{\nabla}(\lambda)$ (resp. $\nabla(\lambda)), \lambda \in \Lambda$, is the projective cover of $\lambda-2(\ell-1) \rho$ as integrable $\tilde{\mathfrak{u}}_{-}^{\sharp}$ (resp. $\left.\mathfrak{u}^{\sharp}-\right)$ module.

(1.6) Because of the isomorphism (1.4) we call $\tilde{\nabla}(\lambda)$ and also by abuse of language $\nabla(\lambda)$ the infinitesimal Verma module of highest weight $\lambda$. By [AJS, 6.3 and 4.10.1]

$$
\tilde{\nabla}(\lambda)(\text { resp. } \nabla(\lambda)) \text { is simple as } \tilde{\mathfrak{u}} \text { - }(\text { resp. } \mathfrak{u}-) \text { module iff } \lambda \equiv(\ell-1) \rho \bmod \ell \Lambda \text {. }
$$

Let $\mathfrak{u}^{++}$(resp. $\mathfrak{u}^{--}$) be the augmentation ideal of $\mathfrak{u}^{+}$(resp. $\mathfrak{u}^{-}$). If $M$ is a $\mathfrak{u}^{ \pm}$-module, let $M^{\mathfrak{u}^{ \pm \pm}}$denote the annihilater of $\mathfrak{u}^{ \pm \pm}$in $M$. By (1.3)

$$
\tilde{\nabla}(\lambda)^{\mathfrak{u}^{++}}=\nabla(\lambda)^{\mathfrak{u}^{++}}=\lambda .
$$

If $\lambda \equiv(\ell-1) \rho \bmod \ell \Lambda$, then $\nabla((\ell-1) \rho+\ell \nu)=\nabla((\ell-1) \rho), \nu \in \Lambda$, is simple, called the Steinberg module, and hence

$$
\nabla((\ell-1) \rho+\ell \nu)^{\mathfrak{u}^{--}}=-(\ell-1) \rho+\ell \nu .
$$

In general, the lowest weight of $\tilde{\nabla}(\lambda)$ (resp. the socle of $\tilde{\nabla}(\lambda)$ ) is $\lambda-2(\ell-1) \rho$ (resp. $w_{0} \lambda^{0}+\ell \lambda^{1}$ if $w_{0}$ is an element of the Weyl group of $R$ such that $w_{0} R^{+}=-R^{+}$and if one writes $\lambda=\lambda^{0}+\ell \lambda^{1}$ with $\lambda^{0} \in \Lambda^{\text {res }}$ and $\lambda^{1} \in \Lambda$ [AJS, 4.2.5]). It follows that

$$
\operatorname{dim} \nabla(\lambda)^{\mathfrak{u}^{--}} \geq 2 \text { unless } \nabla(\lambda) \text { is } \mathfrak{u} \text {-simple. }
$$


(1.7) Let $\mathcal{B}=\mathcal{A} /\left(\phi_{\ell}\right)$ and let $\tilde{\mathfrak{u}}_{\mathcal{B}}$ be the $\mathcal{B}$-subalgebra of $U \otimes_{\mathcal{A}} \mathcal{B}$ generated by $E_{i} \otimes 1, F_{i} \otimes 1$, $K_{i} \otimes 1,\left[\begin{array}{c}K_{i} ; c \\ r\end{array}\right] \otimes 1, i \in[1, n], c \in \mathbb{Z}, r \in \mathbb{N}$. Define its $\mathcal{B}$-subalgebras $\tilde{\mathfrak{u}}_{\mathcal{B}}, \mathfrak{u}_{\mathcal{B}}, \tilde{\mathfrak{u}}_{\mathcal{B}}^{b}$, and $\mathfrak{u}_{\mathcal{B}}^{b}$ as for $\tilde{\mathfrak{u}}$. An infinitesimal Verma module may be defined over $\mathcal{B} ; \tilde{\nabla}_{\mathcal{B}}(\lambda)=\tilde{\mathfrak{u}}_{\mathcal{B}}^{b} \operatorname{Mod}\left(\tilde{\mathfrak{u}}_{\mathcal{B}}, \lambda\right)$, $\lambda \in \Lambda$, admits a structure of $\tilde{\mathfrak{u}}_{\mathcal{B}}$-module like $\tilde{\nabla}(\lambda)$, and we have an isomorphism of $\tilde{\mathfrak{u}}$ modules

$$
\tilde{\nabla}_{\mathcal{B}}(\lambda) \otimes_{\mathcal{B}} \mathcal{K} \simeq \tilde{\nabla}(\lambda)
$$

Restricting the $\tilde{\mathfrak{u}}_{\mathcal{B}}$-action to $\mathfrak{u}_{\mathcal{B}}$, one obtains $\mathfrak{u}_{\mathcal{B}}$-module $\nabla_{\mathcal{B}}(\lambda)=\mathfrak{u}_{\mathcal{B}}^{b} \operatorname{Mod}\left(\mathfrak{u}_{\mathcal{B}}, \lambda\right)$.

Assume now that $\ell$ is a prime $p$. Then $\mathcal{B} / p \mathcal{B}$ is a finite filed $\mathbb{F}_{p}$ of $p$-elements. Let $G$ be a simply connected simple algebraic group over $\mathbb{F}_{p}$ associated to the Cartan matrix $A$ with a Borel subgroup $B$ and a maximal torus $T$ of $B$ both split over $\mathbb{F}_{p}$ such that the roots of $B$ are $-R^{+}$. Let $G_{1}$ (resp. $\left.B_{1}\right)$ be the Frobenius kernel of $G$ (resp. $B$ ). If $\operatorname{Dist}\left(G_{1}\right)$ (resp. Dist $\left(B_{1}\right)$ ) is the algebra of distibutions of $G_{1}$ (resp. $B_{1}$ ), there are isomorphisms of $\mathbb{F}_{p}$-algebras [L2]

$$
\mathfrak{u}_{\mathcal{B}} /\left(K_{i}-1 \mid i \in[1, n]\right) \otimes_{\mathcal{B}} \mathbb{F}_{p} \simeq \operatorname{Dist}\left(G_{1}\right), \quad \mathfrak{u}_{\mathcal{B}}^{b} /\left(K_{i}-1 \mid i \in[1, n]\right) \otimes_{\mathcal{B}} \mathbb{F}_{p} \simeq \operatorname{Dist}\left(B_{1}\right),
$$

and each $\tilde{\nabla}_{p}(\lambda):=\tilde{\nabla}_{\mathcal{B}}(\lambda) \otimes_{\mathcal{B}} \mathbb{F}_{p}, \lambda \in \Lambda$, admits a structure of $G_{1} T$-module (cf. [], II.9]):

$$
\tilde{\nabla}_{p}(\lambda) \simeq \operatorname{Dist}\left(G_{1}\right) \otimes_{\operatorname{Dist}\left(B_{1}\right)}(\lambda-2(p-1) \rho) .
$$

Likewise $\nabla_{\mathcal{B}}(\lambda) \otimes_{\mathcal{B}} \mathbb{F}_{p}$ yields a $G_{1}$-module, which we will denote by $\nabla_{p}(\lambda)$.

\section{$2^{\circ}$ Maximal cyclic modules}

In this section we assume that our Cartan matrix is of type $\mathrm{A}_{n}$. Then all $d_{i}=1$, and we will suppress $i$ from []$_{i}$. By properly specializing the parametres a maximal cyclic module $\mathcal{V}$ for $U_{\zeta}$ of [DJMM] factors through $\mathfrak{u}$, having a unique, up to scalar, $\mathfrak{u}^{+}$-primitive vector. Thus $\mathcal{V}$ is of dimension $\ell^{\left|R^{+}\right|}$and has a simple $\mathfrak{u}$-socle, inviting us to compare $\mathcal{V}$ with infinitesimal Verma modules.

(2.1) Fix $\lambda \in \Lambda$. We define as we may $\mathcal{V}$ to be a $\mathcal{K}$-linear space of basis $u_{\mathbf{m}}, \mathbf{m} \in(\mathbb{Z} / \ell \mathbb{Z})^{\left|R^{+}\right|}$. After $\mathbb{\mathbb { N }}$ we reindex $R^{+}$by the pairs $(i, j), 1 \leq i \leq j \leq n$, and we will denote the $(i, j)$ component of $\mathbf{m}$ by $m_{i j}$. Then $\mathcal{V}$ admits a structure of integrable $\mathfrak{u}$-module as proved in $\mathbb{N}, 5.2]$ such that for each $i \in[1, n]$ and $\mathbf{m} \in(\mathbb{Z} / \ell \mathbb{Z})^{\left|R^{+}\right|}$

$$
\begin{aligned}
E_{i} u_{\mathbf{m}}= & \sum_{k=i}^{n}\left[m_{i k}+m_{i, k-1}-m_{i-1, k-1}-m_{i+1, k}\right] u_{\mathbf{m}+\epsilon(i, k)+\cdots+\epsilon(i, n)}, \\
F_{i} u_{\mathbf{m}}= & \sum_{k=1}^{i}\left[-\lambda_{i}+m_{i+1-k, n-k}-m_{i+1-k, n+1-k}+m_{i-k, n+1-k}-m_{i-k, n-k}\right] \\
& u_{\mathbf{m}-\epsilon(i+1-k, n+1-k)-\epsilon(i+2-k, n+2-k)-\cdots-\epsilon(i, n)}, \\
K_{i} u_{\mathbf{m}}= & \zeta^{\lambda_{i}+2 m_{i n}-m_{i-1, n}-m_{i+1, n}} u_{\mathbf{m}},
\end{aligned}
$$


where $[r]=\frac{\zeta^{r}-\zeta^{-r}}{\zeta-\zeta^{-1}}, \epsilon(i, j) \in(\mathbb{Z} / \ell \mathbb{Z})^{\left|R^{+}\right|}$such that $\epsilon(i, j)_{s t}=\delta_{i s} \delta_{j t}$ for each $s$ and $t$, and any meaningless terms in the sums should be read as 0 . As the structure of $\mathfrak{u}$-module on $\mathcal{V}$ depends on $\lambda$, to be precise, we will denote the $\mathfrak{u}$-module $\mathcal{V}$ by $\mathcal{V}(\lambda)$.

A main theorem of $[\mathrm{N}]$ is that $\mathcal{V}(\lambda)$ has a unique, up to $\mathcal{K}^{\times}, \mathfrak{u}^{+}$-primitive element, i.e.,

$$
\mathcal{V}(\lambda)^{\mathfrak{u}^{++}}=\mathcal{K} u_{\overrightarrow{0}} \quad \text { with } \quad \overrightarrow{0}=(0, \ldots, 0),
$$

and hence by Engel's theorem

$$
\mathcal{V}(\lambda) \text { has a simple } \mathfrak{u} \text {-socle generated by } u_{\overrightarrow{0}} \text {. }
$$

It also follows from (1.3) by dimension that

Proposition. There is an isomorphism of $\mathfrak{u}^{\sharp}$-modules $\mathcal{V}(\lambda) \simeq \nabla(\lambda)$.

(2.2) Recall anitiautomorphism $\tau$ of $\mathfrak{u}$ such that

$$
E_{i} \mapsto F_{i}, \quad F_{i} \mapsto E_{i}, \quad K_{i} \mapsto K_{i}, \quad \forall i \in[1, n]
$$

If $M$ is a $\mathfrak{u}$-module, let $M^{\tau}$ be the $\mathcal{K}$-dual space of $M$ with a $\mathfrak{u}$-action given by

$$
x f=f(\tau(x) ?), \quad f \in M^{*}, x \in \mathfrak{u} .
$$

Then the isomorphism of $\mathfrak{u}^{\sharp}$-modules $\mathcal{V}(\lambda) \simeq \nabla(\lambda)$ from (2.1) yields an isomorphism of $\mathfrak{u}^{-}$-modules

$$
\begin{aligned}
\mathcal{V}(\lambda)^{\tau} & \simeq \nabla(\lambda)^{\tau} \\
& \simeq\left\{\bar{U}_{\zeta} \otimes_{\bar{U}_{\zeta}^{b}}(\lambda-2(\ell-1) \rho)\right\}^{\tau} \quad \text { by }(1.4) \\
& \simeq \bar{U}_{\zeta} \otimes_{\bar{U}_{\zeta}^{\sharp}} \lambda \quad \text { by }[\text { AJS, } 4.10] \\
& \simeq \mathfrak{u}^{-},
\end{aligned}
$$

where $\bar{U}_{\zeta}^{\sharp}$ is the $\mathcal{K}$-subalgebra of $\bar{U}_{\zeta}$ generated by all $E_{\alpha}, \alpha \in R^{+}$, and $K_{i}, i \in[1, n]$. It follows from $[\mathrm{X}, 2.5]$ again that $\mathcal{V}(\lambda)^{\tau}$ has a unique, up to $\mathcal{K}^{\times}, \mathfrak{u}^{-}$-primitive vector, and hence

Corollary. $\mathcal{V}(\lambda)$ has the same simple $\mathfrak{u}$-socle and the same simple $\mathfrak{u}$-head head as $\nabla(\lambda)$.

(2.3) We find, moreover, that

Theorem. The $\mathfrak{u}$-module $\mathcal{V}(\lambda)$ has a unique, up to $\mathcal{K}^{\times}, \mathfrak{u}^{-}$-primitive vector $u_{\mathbf{m}}$ with

$$
m_{i j} \equiv-\sum_{s=1}^{i} \sum_{t=i}^{j} \lambda_{n+s-t} \quad \bmod \ell \quad \forall i \leq j,
$$

which has $\mathfrak{u}^{0}$-weight $w_{0} \lambda$. 
Proof: The argument is the same as for (2.1.4) from [N, 4.2]; let

$$
\sum_{\mathbf{m} \in(\mathbb{Z} / \ell \mathbb{Z})^{\mid R^{+}}} c_{\mathbf{m}} u_{\mathbf{m}} \in \mathcal{V}(\lambda)^{\mathfrak{u}^{--}}, \quad c_{\mathbf{m}} \in \mathcal{K} .
$$

As $F_{i} \sum c_{\mathbf{m}} u_{\mathbf{m}}=0$ for all $F_{i}$, we obtain successively $c_{\mathbf{m}}=0$ unless

$$
-\lambda_{i}+m_{i+1-k, n-k}-m_{i+1-k, n+1-k}+m_{i-k, n+1-k}-m_{i-k, n-k} \equiv 0 \quad \bmod \ell
$$

for each $i$ and $k \in[1, i]$. If $\sum c_{\mathbf{m}} u_{\mathbf{m}} \neq 0$, the system (1) of equations determines $\mathbf{m}$ with $c_{\mathbf{m}} \neq 0$ uniquely as asserted.

(2.4) Corollary. Let $\lambda \in X$.

(i) There is an isomorphism of $\mathfrak{u}^{b}$-modules

$$
\mathcal{V}(\lambda) \simeq \bar{U}_{\zeta} \otimes_{\bar{U}_{\zeta}^{\sharp}}\left(w_{0} \lambda+2(\ell-1) \rho\right) .
$$

(ii) The $\mathfrak{u}$-module $\mathcal{V}(\lambda)$ lifts to an integrable $\tilde{\mathfrak{u}}$-module iff $\lambda \equiv(\ell-1) \rho \bmod \ell \Lambda$. In particular, unless $\lambda \equiv(\ell-1) \rho \bmod \ell \Lambda, \mathcal{V}(\lambda)$ is not isomorphic as $\mathfrak{u}$-module to any infinitesimal Verma module.

Proof: For (i) argue as in (2.1) and (2.2).

(ii) If $\lambda \equiv(\ell-1) \rho \bmod \ell \Lambda$, the simple $\mathfrak{u}$-socle of $\mathcal{V}(\lambda)$ has dimension $\ell^{\left|R^{+}\right|}=\operatorname{dim} \mathcal{V}(\lambda)$ and hence the assertion follows. We may therefore assume that $\lambda \not \equiv(\ell-1) \rho \bmod \ell \Lambda$.

Just suppose $\mathcal{V}(\lambda)$ lift to an integrable $\tilde{\mathfrak{u}}$-module. Then we would have from (1.3) an isomorphism of $\tilde{\mathfrak{u}}^{\sharp}$-modules

$$
\mathcal{V}(\lambda) \simeq \tilde{\nabla}(\lambda+\ell \nu) \quad \text { for some } \nu \in \Lambda
$$

Then the unique $\mathfrak{u}^{-}$-primitive in $\mathcal{V}(\lambda)$ should have by $(1.4)$ weight $\lambda+\ell \nu-2(\ell-1) \rho$. That would yield, arguing as in (2.3), an isomorphism of $\tilde{\mathfrak{u}}^{b}$-modules

$$
\mathcal{V}(\lambda) \simeq \bar{U}_{\zeta} \otimes_{\bar{U}_{\zeta}^{\sharp}}(\lambda+\ell \nu) .
$$

Then $\mathcal{V}(\lambda)=\mathfrak{u}^{-} u_{\overrightarrow{0}}$ as $u_{\overrightarrow{0}}$ is a highest weight vector of $\mathcal{V}(\lambda)$. But $\mathfrak{u}^{-} u_{\overrightarrow{0}}=\mathfrak{u} u_{\overrightarrow{0}}$ is the simple socle of $\mathcal{V}(\lambda)$ and of dimension $<\operatorname{dim} \mathcal{V}(\lambda)$ by (1.6.1), absurd.

(2.5) Assume now that $\ell$ is a prime $p$ and let $\mathcal{B}=\mathcal{A} /\left(\phi_{p}\right)=\mathbb{Z}[v] /\left(\phi_{p}\right)$ as in (1.7). By construction $\mathcal{V}(\lambda)$ may be defined over $\mathcal{B}$ : let $\mathcal{V}_{\mathcal{B}}(\lambda)$ be the free $\mathcal{B}$-module of basis $u_{\mathbf{m}}$, $\mathbf{m} \in(\mathbb{Z} / p \mathbb{Z})^{\left|R^{+}\right|}$, with the $\mathfrak{u}_{\mathcal{B}}$-action given by (2.1.1-3). Regarding $\mathbb{F}_{p}$ as the quotient $\mathcal{B} / p \mathcal{B}=\mathcal{B} /(v-1)$, let $\mathcal{V}_{p}(\lambda)=\mathcal{V}_{\mathcal{B}}(\lambda) \otimes_{\mathcal{B}} \mathbb{F}_{p}$. Then $\mathcal{V}_{p}(\lambda)$ is naturally a $G_{1}$-module for $G=\mathrm{SL}_{n+1} \otimes_{\mathbb{Z}} \mathbb{F}_{p}$ in the setup of (1.7). The proof of (2.1.4) from $\mathbb{N}$ and the argument of (2.3) carry over to obtain

Theorem. Let $\lambda \in \Lambda$

(i) There is an isomorphism of $B_{1}^{+}$-modules $\mathcal{V}_{p}(\lambda) \simeq \nabla_{p}(\lambda)$. 
(ii) $\mathcal{V}_{p}(\lambda)$ has a unique, up to $\mathbb{F}_{p}^{\times}, B_{1}^{+}$- and $B_{1}$-invariant vector, respectively, and hence has a simple $G_{1}$-socle and a simple $G_{1}$-head, where $B_{1}^{+}$is the Frobenius kernel of the Borel subgroup $B^{+}$of $G$ opposite to $B$.

(iii) If $\lambda \not \equiv(p-1) \rho \bmod p \Lambda$, the structure of $G_{1}$-module on $\mathcal{V}_{p}(\lambda)$ does not lift to $G_{1} T$ module, and hence $\mathcal{V}_{p}(\lambda)$ is not isomorphic to any infinitesimal Verma module as $G_{1}$-module.

(2.6) Remark. If the simple $\mathfrak{u}$-module generated by $u_{\overrightarrow{0}}$ in $\mathcal{V}(\lambda)$ and the simple $G_{1}$-module generated by $u_{\overrightarrow{0}} \otimes 1$ in $\mathcal{V}_{\mathcal{B}}(\lambda) \otimes_{\mathcal{B}} \mathbb{F}_{p}$ have the same dimension, then Lusztig's conjecture for the irreducible $\mathrm{SL}_{n+1} \otimes_{\mathbb{Z}} \mathbb{F}_{p}$-modules will follow from [KL] and [KT], [G].

\section{References}

[AJS] Andersen, H. H., Jantzen, J. C. and Soergel, W., Representations of quantum groups at a p-th root of unity and of semisimple groups in characteristics $p$ : Independence of p, Astérisque 220 ( 1994), 1-320.

[APW] Andersen, H. H., Polo, P. and Wen, K., Injective modules for quantum algebras, Am. J. Math. 114 (1992), 571-604.

[C] Casian, L., Proof of the Kazhdan-Lusztig conjecture for Kac-Moody algebras (the characters $\left.\operatorname{ch} L_{w \rho-\rho}\right)$, Adv. Math. 119 (1996), 207-281.

[DJMM] Date, E., Jimbo, M., Miki, K. and Miwa, T., Cyclic representations of $U_{q}(\mathfrak{s l}(n+$ $1, \mathbb{C})$ ) at $q^{N}=1$, Publ. RIMS 27 (1991), 347-366.

[DK] De Concini, C. and Kac, V., Representations of quantum groups at roots of 1, pp. 471-506 in: A. Conne et al. (eds.), Operator Algebras, Unitary Representations, Enveloping Algebras, and Invariant Theory (Colloque Dixmier), Proc. Paris 1989 (Progress in Mathematics 92), Boston etc. 1990 (Birkhäuser)

[H] Humphreys, J. E., Modular representations of classical Lie algebras and semisimple groups, J. Alg. 19 (1971), 51-79.

[J] Jantzen, J. C., Representations of Algebraic Groups, Pure and Applied Mathematics 131, Academic Press, Boston etc. 1987.

[KT] Kashiwara, M. and Tanisaki, T., Kazhdan-Lusztig conjecture for affine Lie algebras with negative level, Duke Math. J. 77 (1995), 21-62

[KL] Kazhdan, D.A. and Lusztig, G., Tensor structures arising from affine Lie algebras I, II, J. AMS 6 (1993), 905-1011; Tensor structures arising from affine Lie algebras III, IV, J. AMS 7 (1994), 335-453.

[L1] Lusztig, L., Finite dimensional Hopf algebras arising from quantized universal enveloping algebras, J. AMS. 3 (1990), 257-296.

[L2] Lusztig, L., Quantum groups at roots of 1, Geom. Ded. 35 (1990), 89-114.

[N] Nakashima, T., Irreducible modules of finite dimensional quantum algebras of type $A$ at roots of unity, J. Math. Phys. 43 (2002), no. 4, 2000-2014. 
[X] Xi, N., Irreducible modules of quantized enveloping algebras at roots of 1 , Publ. RIMS 32 (1996), 235-276. 